\title{
Analysis of Risk Factors of Postoperative Neurological Complications in Patients with Stanford Type A Aortic Dissection Undergoing Sun's Procedure
}

\author{
Hui Zhang*, Ruicheng Zhang, Hua Zhang, Feng Li, Jianming Zhao, Shen Ma, Rujun Zhu, Pingfan Wang \\ Department of Cardiac Surgery,Henan Provincial Chest Hospital,Zhengzhou 450000, Henan Province, China
}

*Corresponding author: Hui Zhang, 471534281@qq.com

\begin{abstract}
Objective: This study analyzed the risk factors of neurological complications in patients with Stanford type A aortic dissection after Sun's procedure in a single-center with the purpose of improving the effects. Methods: From January 2019 to December 2020, the clinical data of 480 patients with Stanford type A aortic dissection, who were treated by Sun's procedure in our center were retrospectively analyzed. Univariate and multivariate logistic regression analyses were used to determine the risk factors of postoperative neurological complications. According to whether there were neurological complications after surgery, they were divided into two groups: the group with complications $(\mathrm{n}=70)$ and the group without complications $(\mathrm{n}=410)$. The clinical data of the two groups were collected and compared. Results: There were 70 cases of patients with postoperative neurological complications in 480 cases. The incidence rates of temporary neurological dysfunction (TND) and permanent neurological dysfunction (PND) was 11.5\% (55/480) and 3.1\% (15/480), respectively. Univariate analysis showed that the age ( $\geq 70$ years), stroke history, femoral artery intubation and cardiopulmonary bypass (CPB) time were associated with postoperative PND $(\mathrm{p}<0.05)$. Renal dysfunction, emergency surgery, postoperative hypernatremia, postoperative hyperglycemia, postoperative hypoxemia, postoperative low cardiac output syndrome, and assisted time of suction influenced the occurrence of postoperative TND ( $p<0.05$ ). Multivariate logistic regression analysis showed that age ( $\geq 70$ years), stroke history, femoral artery intubation and CPB time were independent risk factors for PND. Renal dysfunction, emergency surgery, postoperative hypernatremia, postoperative hyperglycemia, postoperative hypoxemia, postoperative low cardiac output syndrome, and aspiration time were independent risk factors for TND. Compared with the two groups, the hospitalization time and ICU time of the patients in the neurological complications group were significantly prolonged, and the mortality rate was significantly increased $(\mathrm{p}<0.05)$. Conclusion: There are many risk factors for neurological complications in patients with Stanford type A aortic dissection after surgery. With the improvement of surgical techniques, optimization of cerebral perfusion, and interventions for risk factors, Sun's procedure remains the preferred treatment for Stanford type A aortic dissection.
\end{abstract}

Keywords: Aortic dissection; Neurological complications; Sun's procedure; Risk factors

Publication date: November 2021; Online publication: November 30, 2021

\section{Introduction}

Stanford type A aortic dissection is the most dangerous and serious condition in the cardiac surgery department. This condition is associated with rapid progression and high mortality. Along with the enhanced recognition of and importance placed on acute chest pain, the incidence rate and diagnosis rate of Stanford type A aortic dissection in China are increasing every year ${ }^{[1]}$. Sun's procedure is the one of the most effective treatments ${ }^{[2]}$. Since the processing of aortic arch replacement under deep hypothermic 
circulatory arrest (DHCA) is needed, nervous system compatibility not only affects the clinical effect of Sun's procedure, but also reduces the quality of life of patients and even endangers their lives ${ }^{[3]}$. How to reduce nervous system compatibility has gradually become a research hotspot in the field of angiocarpy. The present study retrospectively analyzed the clinical data derived from patients with Stanford type A dissection who had undergone Sun's procedure. The related risk factors of postoperative nervous system compatibility are also discussed in this paper.

\section{Materials and methods}

\subsection{Research subjects}

This study was reviewed and approved by the hospital medical ethics committee, and the patients and their families signed the informed consent form. The data of 480 patients with Stanford type A aortic dissection treated by Sun's procedure in our center from January 2019 to December 2020 were analyzed retrospectively. They were diagnosed by computed tomographic angiography (CTA) of the aorta and Doppler ultrasound before operation, and Sun's procedure was performed within two weeks. Patients who died of large-area cerebral infarction before operation or who were in coma, patients who died within 48 hours after operation and subjects with incomplete clinical data were excluded from this study ${ }^{[4]}$.

\subsection{Surgical methods}

All operations were performed under intravenous-inhalation combined anesthesia, DHCA and selective cerebral perfusion. The cannula sites for establishing cardiopulmonary bypass are divided into femoral artery and right axillary artery. The right axillary artery, innominate artery, left common carotid artery and left subclavian artery can be the cannula sites for selective cerebral perfusion. Aortic root operation can be divided into simple ascending aortic replacement, aortic valve junction suspension "sandwich" integrated angioplasty plus ascending aortic replacement, Bentall method, modified David method, Cabrol method and Wheat method. Sun's procedure was performed on the aortic arch and distal end. Intraoperative intravenous administration of hormone, combined with ice cap physical cooling and brain oxygen monitoring for brain protection, was performed. Patients with postoperative neurological complications were given dehydration, hormones, neurotrophic drugs and hyperbaric oxygen treatment when necessary.

\subsection{Relevant indicators}

Preoperative indexes include age, body mass index, hypertension history, history of coronary heart disease, history of stroke, history of diabetes, medical history of chronic obstructive pulmonary disease (COPD), history of renal function insufficiency, emergency surgery, etc. Intraoperative indexes include the arterial intubation site for establishing cardiopulmonary bypass (CPB), selective cerebral perfusion intubation site, CPB time, operation time, aortic occlusion time and DHCA time. Postoperative indexes included low cardiac output syndrome, hypoxemia, blood glucose and blood sodium level, ventilator assistance time, ICU time and hospitalization time. Hypernatremia was defined as serum sodium $>150 \mathrm{mmol} / \mathrm{L}$ after two postoperative consecutive reviews.

\subsection{Diagnostic criteria for neurological complications}

Postoperative cerebral nervous system complications, including temporary neurological dysfunction (TND) and permanent neurological dysfunction (PND), were confirmed by imaging examination and neurophysicians. TND refers to neurological dysfunction such as delayed awakening or transient clouding of consciousness, delirium, indifference and confusion after operation, which is mainly related to cerebral ischemic injury caused by blood flow interruption or low flow perfusion and other factors. There is no positive expression on brain magnetic resonance imaging (MRI) or computed tomography (CT), and it can 
return to normal before discharge or in a short time after symptomatic treatment. PND refers to stroke caused by embolism or bleeding after operation, which has positive manifestations of brain MRI or CT. Recovery of neurological function is difficult after treatment, thereby affecting the prognosis of patients.

\subsection{Statistical methods}

SPSS 20.0 statistical software was used for data processing. The measurement data were expressed in mean \pm standard deviation, and two independent sample was analyzed using t-test. Count data analysis was tested by $\chi^{2}$ test. The influencing factors of postoperative nervous system compatibility were analyzed by univariate and multivariate logistic regression. If $\mathrm{P}<0.05$, the difference was statistically significant.

\section{Results}

\subsection{Occurrence of postoperative neurological complications}

Out of 480 cases, there were 70 cases of Stanford type A aortic dissection with neurologic complications after Sun's procedure, with an incidence rate of 14.6\%, including 15 PND cases with an incidence rate of $3.1 \%(15 / 480)$ and 70 TND cases with an incidence rate of $11.5 \%(55 / 480)$.

\subsection{Risk factor analysis results of PND}

Univariate analysis showed that there were significant differences in the elderly ( $\geq 70$ years old), stroke history, femoral artery intubation and CPB time between PND group and non-PND group (P $<0.05)$, as shown in Table 1. Multivariate logistic regression analysis showed that the risk factors of PND after Sun's procedure for Stanford type A aortic dissection were old age ( $\geq 70$ years), history of stroke, femoral artery intubation and CPB time, and the difference was statistically significant ( $<<0.05)$, as shown in Table 2.

Table 1. PND univariate analysis results

\begin{tabular}{lcccc}
\hline Variable & $\begin{array}{c}\text { PND group } \\
(\mathbf{1 5} \text { cases) }\end{array}$ & $\begin{array}{c}\text { Non-PND group } \\
\text { (465 cases) }\end{array}$ & P value & $\chi^{2} /$ T value \\
\hline Old age (> 70 years) & $6(40 \%)$ & $36(7.7 \%)$ & 0.000 & 12.459 \\
Body mass index & $26.1 \pm 2.7$ & $25.9 \pm 2.5$ & 0.501 & 0.584 \\
Diabetes & $2(13.3 \%)$ & $37(8.0 \%)$ & 0.499 & 0.457 \\
Hypertension & $9(60 \%)$ & $255(54.8 \%)$ & 0.833 & 0.044 \\
Stroke & $3(20 \%)$ & $14(3.0 \%)$ & 0.001 & 9.92 \\
COPD & $1(6.6 \%)$ & $30(6.5 \%)$ & 0.975 & 0.001 \\
Coronary heart disease & $2(13.3 \%)$ & $46(9.9 \%)$ & 0.696 & 0.152 \\
Emergency surgery & $8(53.3 \%)$ & $255(54.8 \%)$ & 0.949 & 0.004 \\
Renal insufficiency & $2(13.3 \%)$ & $36(7.7 \%)$ & 0.476 & 0.507 \\
Femoral artery intubation & $6(40 \%)$ & $46(9.9 \%)$ & 0.003 & 8.759 \\
Aortic occlusion time & $85.7 \pm 10.3$ & $87.3 \pm 9.1$ & 0.238 & 0.942 \\
DHCA time & $20.8 \pm 2.4$ & $21.1 \pm 1.8$ & 0.487 & 0.621 \\
CPB time & $214 . \pm 42.3$ & $187.3 \pm 16.7$ & 0.032 & 7.429 \\
Postoperative hyperglycemia & $2(13.3 \%)$ & $35(7.5 \%)$ & 0.453 & 0.562 \\
Postoperative hypernatrium & $1(6.7 \%)$ & $24(5.2 \%)$ & 0.808 & 0.059 \\
Postoperative hypoxemia & $5(33.3 \%)$ & $154(33.1 \%)$ & 1.000 & 0.000 \\
Postoperative low cardiac output syndrome & $1(6.7 \%)$ & $33(7.1 \%)$ & 0.949 & 0.004 \\
Ventilator-assisted time & $60.6 \pm 33.4$ & $59.5 \pm 30.6$ & 0.743 & 0.659 \\
\hline
\end{tabular}


Table 2. Results of PND multivariate logistic regression analysis

\begin{tabular}{lccccc}
\hline Risk factor & $\begin{array}{c}\text { Regression } \\
\text { coefficient }\end{array}$ & $\begin{array}{c}\text { Standard } \\
\text { Error }\end{array}$ & P value & Odds ratio & $\begin{array}{c}\text { 95\% confidence } \\
\text { interval }\end{array}$ \\
\hline Old age (> 70 years) & 0.855 & 0.832 & 0.031 & 1.745 & $1.059-2.907$ \\
Stroke & 1.217 & 0.389 & 0.000 & 2.847 & $1.638-4.951$ \\
CPB time & 3.066 & 0.587 & 0.001 & 3.119 & $1.322-8.021$ \\
Femoral artery intubation & 2.147 & 0.672 & 0.001 & 3.263 & $5.502-15.238$ \\
\hline
\end{tabular}

\subsection{Risk factor analysis results of TND}

Univariate analysis showed that there was significant difference between TND group and non-TND group in patients with renal dysfunction, emergency surgery, postoperative high blood glucose, postoperative hypernatremia, postoperative hypoxemia, low cardiac output syndrome and ventilator assistance time $(\mathrm{P}<$ 0.05), as shown in Table 3. The results of multivariate logistic regression analysis showed that the risk factors of TND after Sun's procedure for Stanford type A aortic dissection were combined with renal function insufficiency, emergency surgery, postoperative high blood glucose, postoperative hypernatremia, postoperative hypoxemia, postoperative low cardiac output syndrome and ventilator assistance time. The difference was statistically significant $(\mathrm{P}<0.05)$, as shown in Table 4.

\subsection{Comparison of clinical outcomes between complication group and non-complication group}

Compared with the non-complication group, the hospitalization time and ICU time in the nervous system complication group were significantly longer, and the hospitalization mortality was significantly higher (P $<0.05)$, as shown in Table 5.

Table 3. Results of TND single factor analysis

\begin{tabular}{lcccc}
\hline Variable & $\begin{array}{c}\text { TND group } \\
(\mathbf{1 5} \text { cases) }\end{array}$ & $\begin{array}{c}\text { Non-TND group } \\
\mathbf{( 4 2 5} \text { cases) }\end{array}$ & P value & $\chi^{2} /$ T value \\
\hline Old age (> 70 years) & $5(9.1 \%)$ & $42(9.9 \%)$ & 0.864 & 0.029 \\
Body mass index & $27.3 \pm 3.7$ & $26.5 \pm 4.9$ & 0.695 & 7.326 \\
Diabetes & $5(9.1 \%)$ & $43(10.1 \%)$ & 0.828 & 0.047 \\
Hypertension & $22(40 \%)$ & $192(45.2 \%)$ & 0.648 & 0.208 \\
Stroke & $2(3.6 \%)$ & $16(3.8 \%)$ & 0.964 & 0.002 \\
COPD & $3(5.5 \%)$ & $25(5.9 \%)$ & 0.905 & 0.014 \\
Coronary heart disease & $6(10.9 \%)$ & $38(8.9 \%)$ & 0.666 & 0.186 \\
Emergency surgery & $45(81.8 \%)$ & $214(50.3 \%)$ & 0.025 & 5.032 \\
Renal insufficiency & $10(18.2 \%)$ & $34(8.0 \%)$ & 0.029 & 4.714 \\
Femoral artery intubation & $6(10.9 \%)$ & $41(9.6 \%)$ & 0.788 & 0.072 \\
Aortic occlusion time & $84.3 \pm 11.9$ & $87.5 \pm 7.3$ & 0.562 & 0.085 \\
DHCA time & $23.4 \pm 5.1$ & $22.1 \pm 4.5$ & 0.238 & 1.188 \\
CPB time & $207.5 \pm 33.8$ & $194.3 \pm 35.7$ & 0.569 & 0.954 \\
Postoperative hyperglycemia & $10(18.2 \%)$ & $28(6.6 \%)$ & 0.007 & 7.083 \\
Postoperative hypernatrium & $6(10.9 \%)$ & $8(1.9 \%)$ & 0.000 & 12.391 \\
Postoperative hypoxemia & $10(18.2 \%)$ & $18(4.2 \%)$ & 0.000 & 13.950 \\
Postoperative low cardiac output syndrome & $10(18.2 \%)$ & $26(6.1 \%)$ & 0.004 & 8.101 \\
Ventilator-assisted time & $69.5 \pm 42.1$ & $55.3 \pm 31.4$ & 0.001 & 12.614 \\
\hline
\end{tabular}


Table 4. Results of TND multivariate logistic regression analysis

\begin{tabular}{lccccc}
\hline Risk factor & $\begin{array}{c}\text { Regression } \\
\text { coefficient }\end{array}$ & $\begin{array}{c}\text { Standard } \\
\text { Error }\end{array}$ & P value & $\begin{array}{c}\text { Odds ratio } \\
\text { 95\% confidence } \\
\text { interval }\end{array}$ \\
\hline Renal insufficiency & 2.536 & 0.663 & 0.002 & 10.17 & $2.641-13.108$ \\
Emergency surgery & 1.476 & 0.625 & 0.020 & 4.375 & $1.284-14.264$ \\
Postoperative hypernatrium & 1.218 & 0.573 & 0.024 & 3.531 & $1.203-8.527$ \\
Postoperative hyperglycemia & 0.341 & 0.165 & 0.024 & 3.51 & $1.143-7.137$ \\
Postoperative low cardiac output syndrome & 2.032 & 0.672 & 0.022 & 3.125 & $1.173-9.287$ \\
Postoperative hypoxemia & 1.061 & 0.323 & 0.002 & 2.26 & $1.401-6.732$ \\
Ventilator-assisted time & 1.116 & 0.626 & 0.023 & 4.32 & $1.363-12.595$ \\
\hline
\end{tabular}

Table 5. Comparison of clinical outcomes between the two groups

\begin{tabular}{lcccc}
\hline Group & Number of cases & Length of stay (days) & ICU time (hours) & Inpatient mortality (\%) \\
\hline Complication group & 70 & $23.4 \pm 7.1$ & $263.4 \pm 125.1$ & $11(15.7 \%)$ \\
Non-complication group & 410 & $14.1 \pm 5.6$ & $123.4 \pm 65.1$ & $16(3.9 \%)$ \\
$\chi^{2} /$ T value & & 7.238 & 10.452 & 13.03 \\
P value & & 0.02 & 0.01 & 0.000 \\
\hline
\end{tabular}

\section{Discussion}

Stanford type A aortic dissection has many risk factors, including hypertension and blood flow shear force. It leads to the rupture of aortic intima and involves the aortic arch. It is a serious life-threatening angiocarpy disaster. The mortality within 24 hours without surgical treatment increased by $1-2 \%$ per hour, and the mortality within 1 week was more than $70 \%{ }^{[5-6]}$. Aortic arch reconstruction in Sun's procedure requires DHCA, selective cerebral perfusion to provide a blood-free operation area, and complete brain protection measures. The operation involved is complicated, and the operation time and cardiopulmonary bypass time are long. In addition, this may lead to many postoperative complications. Neurological complication is one of the main complications, which seriously affects the treatment effect and patient prognosis ${ }^{[7-8]}$. Sun's procedure has been used in our hospital for 15 years, and we have seen a gradual increase in the average annual number of operations. The purpose of this study is to analyze the risk factors of nervous system complications after Sun's procedure for Stanford type A aortic dissection, so as to improve the treatment effect.

At present, the research results of different centers at home and abroad are different. The research of Naito et al. showed that the total incidence rate of postoperative nervous system complication was $20.3 \%$, of which TND and PND accounted for $12.6 \%$ and $7.7 \%$, respectively ${ }^{[9]}$. Local study reported that the incidence of early postoperative neurological complications was $14.7 \%$, including $11.5 \%$ for TND and $3.2 \%$ for PND ${ }^{[10]}$. In this study, the incidence rates of postoperative TND and PND were $11.5 \%$ and $3.1 \%$, respectively. Compared with other local and foreign studies, the present study may have benefited from the surgical skill proficiency of our medical staff and their teamwork.

The mechanism of neurological complications after Sun's operation for Stanford type A aortic dissection is not completely clear. We believe that it is related to the influence of dissection on blood supply to the brain, as well as a variety of factors before, during and after the operation. This study shows that the independent risk factors for postoperative PND are as follows: (1) Old age ( $\geq 70$ years) and history of preoperative stroke. Older age, especially higher than 70 years old, increases the risk for cerebrovascular 
atherosclerosis that leads to hemadostenosis and even stroke. On the other hand, the elderly may suffer from physiological degeneration, reduced self-regulatory cerebrovascular ability, and significantly reduced tolerance to DHCA, which increases the risk for PND after surgery ${ }^{[11]}$. Through comparative analysis, this study concluded that it is crucial to comprehensively evaluate the cerebrovascular condition of the patients before operation, select appropriate brain protection measures, such as selective cerebral perfusion, cerebral oxygen saturation monitoring, shortening of DHCA time, and maintenance of stable perioperative cerebral blood flow. (2) Femoral artery intubation, which was selected to establish CPB. The research results of Liu et al. ${ }^{[12]}$ in China show that the establishment of extracorporeal circulation by femoral artery intubation does not increase the incidence of postoperative nervous system complications. The conclusion of this study is contrary to that of Mariano et al. ${ }^{[13]}$ Researchers in Europe and other places mostly believe that choosing femoral artery intubation to establish CPB increases the risk of plaque shedding or air embolism ${ }^{[14]}$. To analyze the causes according to the actual situation, most of the study participants we selected for this study were over 60 years old. Femoral artery intubation caused atherosclerotic plaque to fall off, resulting in PND. Most of the factors were emergency surgery and improper femoral artery intubation, which causes air embolism or false cavity entry. Insufficient CPB flow and pressure lead to insufficient cerebral perfusion. (3) CPB time. Zhang et al. ${ }^{[15]}$ pointed out that CPB time is an independent risk factor for postoperative neurological complications. This study also proved that in the process of solid external circulation, the tangible components of blood are destroyed, a large number of inflammatory mediators are released, coagulation, fibrinolysis and complement systems are activated, thrombosis, air embolism, atherosclerotic plaque and tissue debris fall off during operation form microemboli, resulting in cerebral circulation embolism and PND ${ }^{[16]}$.

The independent risk factors for postoperative TND are as follows: (1) Emergency surgery. The condition of aortic dissection is urgent, critical and severe. Emergency surgery is often needed to save lives. The critical condition of acute onset and serious systemic inflammatory reaction lead to the corresponding increase in the incidence of postoperative TND. (2) Combined with renal insufficiency (serum creatinine > $185.6 \mu \mathrm{mol} / \mathrm{L}$ ). Studies have found that dissection involving renal artery causes renal ischemia, and subsequently multiple strikes such as surgery, DHCA, systemic inflammatory reaction and ischemiareperfusion injury, resulting in internal environment disorder, accumulation of endotoxin or inflammatory mediators and aggravating cerebral edema ${ }^{[17]}$. (3) Postoperative hyperglycemia and hypernatremia. There is evident that the release of excitatory amino acids glutamate and aspartate increases when the blood glucose increases after operation, having a toxic effect on the nervous system accompanied by ischemia ${ }^{[18]}$. In addition, the increase of blood glucose also aggravates the systemic inflammatory reaction. The incidence rate of postoperative hypernatremia was 1.5\% (7/480). Multivariate logistic regression analysis was an independent risk factor for postoperative TND. The main factors of postoperative hypernatremia were complex preoperative lesions, long time of CPB and DHCA, severe postoperative condition, hyperglycemia and excessive daily average dosage of mannitol. Local researchers believe that the incidence rate of hypernatremia after aortic dissection is as high as $35.3 \%{ }^{[19]}$. According to a retrospective study of patients in the neurological ICU of a foreign university, the incidence rate of postoperative hypernatremia was $7.9 \%$, while the incidence rate of postoperative hypernatremia in patients using dehydration treatment such as mannitol is as high as $24.3 \%$. It is summarized that postoperative hypernatremia is related to mortality, and correcting hypernatremia is conducive to the smooth recovery of patients ${ }^{[20]}$. We found that to avoid postoperative hypernatremia, we should first shorten the time of CPB and DHCA, then, set the dosage of mannitol to within the recommended range, and finally reduce postoperative hyperglycemia in time. However, the independent correlation between hypernatremia and postoperative TND still needs further exploration in large sample research and through observation. (4) Postoperative hypoxemia $\left(\mathrm{PaO}_{2}\right.$ $<60 \mathrm{mmHg}$ ) and ventilator-assisted time. Local studies pointed out that prolonging the auxiliary time of 
ventilator can increase the risk of postoperative TND ${ }^{[21]}$. Through analysis, Cefarelli found that ventilatorassisted time, postoperative hypoxemia and postoperative nervous system compatibility affect one another. The more severe the hypoxemia is, the longer the ventilator-assisted time is, thus increasing the risk of postoperative TND. In turn, postoperative TND prolongs ventilator-assisted time and ICU time, and even further increases mortality ${ }^{[22]}$. Through retrospective analysis, we should take active intervention measures to shorten the time of CPB and DHCA, reduce pulmonary edema, improve oxygenation index, ensure blood and oxygen supply of brain cells and reduce the occurrence of postoperative TND. (5) Postoperative low cardiac output syndrome. The results of this group showed that many organs of the body were in a state of low perfusion ischemia when postoperative low cardiac output occurred. Because brain cells had poor tolerance to ischemia and hypoxia, it was easy to cause neurological complications.

The commonly used methods for brain protection in this study include DHCA and selective cerebral perfusion. According to the involvement of dissection, unilateral cerebral perfusion or bilateral cerebral perfusion is adopted, with an average time of $21.2 \pm 2.4 \mathrm{~min}$. It has the following advantages: (1) Deep hypothermia can reduce the metabolic rate of brain cells, enhance the tolerance of brain cells to hypoxia, and reduce damage to the nervous system ${ }^{[23]}$. (2) Cerebral perfusion within the safe time limit (30-40 min) not only conforms to physiological perfusion, but also alleviates ischemic injury, thus reducing the incidence of neurological complications ${ }^{[24-25]}$. Early reports showed that the combined application of deep hypothermia and selective cerebral perfusion not only effectively prolonged the time of circulatory arrest, but also effectively reduced the incidence of neurological and other complications ${ }^{[26-27]}$.

In conclusion, with the improvement of medical technology, clinical workers should increase the screening frequency on the high-risk groups, comprehensively evaluate the condition, carefully analyze the surgical indications, strengthen the management and intervention of risk factors during the perioperative period, reduce the incidence of postoperative neurological complications and improve the treatment effect of Sun's procedure. Because this study is a single-center retrospective study, the sample size is limited and has certain limitations. Therefore, the independent risk factors need to be further clarified in multi-center, large-sample prospective study.

\section{Disclosure statement}

The authors declare no conflict of interest.

\section{References}

[1] Guo DC, Hostetler EM, Fan Y, et al., 2017, Heritable Thoracic Aortic Disease Genes in Sporadic Aortic Dissection. J Am Coll Cardiol, 70(21): 2728-2730.

[2] Kitamura T, Nie M, Horai T, et al., 2017, Direct True Lumen Cannulation ("Samu-rai" Cannulation) for Acute Stanford Type A Aortic Dissection. Ann Thorac Surg, 104(6): e459-61.

[3] Song XR, Cheng ZY, Liu FR, et al., 2016, Clinical Study of Postoperative Delirium After Stanford Type A Aortic Dissection. Chinese Journal of Thoracic and Cardiovascular Surgery, 32(10): 607-611.

[4] Sun LZ, Ma WG, Zhu JM, et a1., 2013, Sun's Procedure for Chronic Type A Aortic Dissection:Total Arch Replacement Using a Tetrafur Cate Graft with Stented Elephant Trunk Implantation. Ann Cardiothorac Surg, 2(5): 665-666.

[5] Feng WZ, Zhou JQ, Yu GM, et al., 2017, Association of Serum Cystatin C Levels with Mortality in Patients with Acute Type A Aortic Dissection. Oncotarget, 8(60): 101103-101111.

[6] Qi RD, Zhu JM, Chen L, et al., 2017, Observation of Sun's Operation in the Treatment of Chronic Stanford Type B Aortic Dissection Involving the Aortic Arch. National Medical Journal of China, 
97(24): 1867-1870.

[7] Feng XQ, Chen JF, Wu L, et al., 2017, Comparison of Effects of Selective Cerebral Perfusion Combined with Distal Perfusion and Selective Cerebral Perfusion in Administration and Management of Aortic Dissection Cardiopulmonary Bypass. Chinese Journal of Experimental Surgery, 34(10): 1778-1780.

[8] Liao YL, Huang D, Liao YY, et al., 2017, Aortic Dissection Stanford TYPE A Aortic arch Replacement and Descending Aortic Stent Implantation Deep Hypothermia Cardiopulmonary Bypass Anesthesia Management. Shaanxi Medical Journal, 46(8): 1012-1013.

[9] Naito K, Naishida H, Takanashi S, 2016, Permanent Neurological Deficit in Surgical Repair for Acute Type A Aortic Dissection. Kyobu Geka, 69(4): 299-303.

[10] Liu H, Chang Q, Zhang H, et al., 2017, Predictors of Adverse Outcome and Transient Neurological Dysfunction Following Aortic Arch Replacement in 626 Consecutive Patients in China. Heart Lung Circ, 26(2): 172-178.

[11] Chen LW, Dai XF, Wu XJ, et al., 2017, Ascending Aorta and Hemi-Arch Replacement Combined with Modified Triple-Branched Stent Graft Implantation for Repair of Acute DeBakey Type I Aortic Dissection. Ann Thorac Surg, 103(2): 595-601.

[12] Liu H, Chang Q, Zhang HT, et al., 2014, Risk Factors for Neurologic Complications after Acute Stanford Type A Aortic Dissection. Chinese Journal of Thoracic and Cardiovascular Surgery, 30(6): 342-345.

[13] Cefarelli M, Murana G, Surace G, et al. 2017, Elective Aortic Arch Repair: Factors Influencing Neurological Outcome in 791 Patients. Ann Thorac Surg, 104(6): 2016-2023.

[14] De Paulis R, Czerny M, Weltert L, et al., 2015, Current Trends in Cannulation and Neurological Protection During Surgery of the Aortic Arch in Europe. Eur J Cardiothorac Surg, 47(5): 917-923.

[15] Zhang CC, Fa XE, Huang ZF, et al., 2017, Analysis of Risk Factors Related to Nervous System Compatibility after Type A Aortic Dissection. Journal of Southeast University (Medical Science Edition), 36(3): 361-364.

[16] Pan E, Wallinder A, Peterstrom E, et al. 2019, Outcome After Type A Aortic Dissection Repair in Patients with Preoperative Cardiac Arrest. Resuscitation. 144: 1-5.

[17] Hui Z, Guyan W, Lijing Y, et al., 2018, Acute Kidney Injury After Total Arch Replacement Combined with Frozen Elephant Trunk Implantation: Incidence, Risk Factors, and Outcome. Cardiothorac Vasc Anesth, 32(5): 2210-2217.

[18] Matsuzono K, Suzuki M, Arai N, et al., 2018, Successful Tissue Plasminogen Activator for a Patient with Stroke After Stanford Type A Aortic Dissection Treatment. Journal of Stroke and Cerebrovascular Diseases, 27(7): e132-e134.

[19] Fei ZH, Qiu J, Ma DW, et al., 2013, Reason Analysis of Hypernatremia after Deep Hypothermia Circulatory Arrest of Aortic Dissection. Journal of Cardiovascular and Pulmonary Diseases, 32(5): 594-597.

[20] Zhang YZ, Qie JY, Zhang QH, 2015, Incidence and Mortality Prognosis of Dysnatremias in Neurological Critically Ill Patients. Eur Neurol, 73: 29-36.

[21] Fang MX, Xiong WP, Zeng R, et al., 2016, Risk Factors Analysis of Postoperative Delirium in Patients with Stanford Type A Aortic Dissection. Chinese Journal of Clinical Thoracic and Cardiovascular Surger, 23(4): 357-361.

[22] Bartolomeo R, Berretta P, Pantaleo A, et al., 2017, Long-Term Outcomes of Open Arch Repair After a 
Prior Aortic Operation: Our Experience in 154 Patients. Ann Thorac Surg, 103: 1406-1412.

[23] Yang B, Malik A, Waidley V, et al., 2018, Short-Term Outcomes of a Simple and Effective Approach to Aortic Root and Arch Repair in Acute Type A Aortic Dissection. The Journal of Thoracic and Cardiovascular Surgery, 155(4): 1360-1370.

[24] Geube M, Sale S, Svensson L, 2017, Routine Use of Brain Perfusion Techniques is not Supported in Deep Hypothermic Circulatory Arrest. J Cardiothorac Vasc Anesth, 31(5): 1905-1909.

[25] Angeloni E, Melina G, Refice SK, et al., 2015, Unilateral Versus Bilateral Antegrade Cerebral Protection During Aortic Surgery: An Updated Meta - Analysis. Ann Thorac Surg, 99(6): 2024-2031.

[26] Pacini D, Murana G, Dimarco L, et al., 2018, Cerebral Perfusion Issues in Type A Aortic Dissection. J Vis Surg, 4: 77.

[27] Hata M, Sezai A, Yoshitake I, et al., 2010, Clinical Trends in Optimal Treatment Strategy for Type A Acute Aortic Dissection. Ann Thorac Cardiovasc Surg, 16(4): 228-235. 